\title{
Variable Temperature-Scanning Hall Probe Microscopy With GaN/AIGaN Two-Dimensional Electron Gas (2DEG) Micro Hall Sensors in 4.2-425 K Range Using Novel Quartz Tuning Fork AFM Feedback
}

\author{
R. Akram ${ }^{1}$, M. Dede ${ }^{1}$, and A. Oral ${ }^{2}$ \\ ${ }^{1}$ Department of Physics, Bilkent University, 06800 Ankara, Turkey \\ ${ }^{2}$ Faculty of Engineering and Natural Sciences, Sabanci University, 34956 Istanbul, Turkey
}

\begin{abstract}
In this paper, we present the fabrication and variable temperature (VT) operation of Hall sensors, based on GaN/AIGaN heterostructure with a two-dimensional electron gas (2DEG) as an active layer, integrated with quartz tuning fork (QTF) in atomic force-guided (AFM) scanning Hall probe microscopy (SHPM). Physical strength and a wide bandgap of GaN/AlGaN heterostructure makes it a better choice to be used for SHPM at elevated temperatures, compared to other compound semiconductors (AIGaAs/GaAs and InSb), which are unstable due to their narrower bandgap and physical degradation at high temperatures. GaN/AlGaN micro Hall probes were produced using optical lithography and reactive ion etching. The active area, Hall coefficient, carrier concentration, and series resistance of the Hall sensors were $\sim 1 \times 1 \mu \mathrm{m}, 10 \mathrm{~m} \Omega / \mathrm{G}$ at $4.2 \mathrm{~K}, 6.3 \times 10^{12} \mathrm{~cm}^{-2}$ and $12 \mathrm{k} \Omega$ at room temperature and $7 \mathrm{~m} \Omega / \mathrm{G}, 8.9 \times 10^{12} \mathrm{~cm}-2$ and $24 \mathrm{k} \Omega$ at $400 \mathrm{~K}$, respectively. A novel method of AFM feedback using QTF has been adopted. This method provides an advantage over scanning tunneling-guided feedback, which limits the operation of SHPM the conductive samples and failure of feedback due to high leakage currents at high temperatures. Simultaneous scans of magnetic and topographic data at various pressures (from atmospheric pressure to high vacuum) from 4 . to $425 \mathrm{~K}$ will be presented for different samples to illustrate the capability of GaN/AlGaN Hall sensors in VT-SHPM.
\end{abstract}

Index Terms-Atomic force microscopy (AFM), GaN/AIGaN heterostructure, Hall probe, quartz tuning fork (QTF), scanning Hall probe microscopy (SHPM).

\section{INTRODUCTION}

$\mathbf{T}$ THE recent interest in monitoring the localized surface magnetic field fluctuations with high spatial and magnetic field resolutions, at elevated temperatures, has made the scanning Hall probe microscopy (SHPM) [1] one of the best choices as it provides means to perform sensitive, noninvasive, and quantitative imaging. The SHPM technique offers various advantages and complements the other magnetic imaging methods like scanning superconducting quantum interference device microscopy (SSM) [2], magnetic force microscopy (MFM) [3], magnetic near field scanning optical microscopy [4], and Kerr microscopy [5]. However, there have been few reports [6], [7] on magnetic imaging with Hall sensors at the high temperature regime. The main reason behind the unsuitability of SHPM at high temperatures is the use of compound semiconductors such as $\mathrm{AlGaAs} / \mathrm{GaAs}$ and InSb for fabrication of Hall probes, which are unstable at elevated temperatures due to their narrow bandgap and physical degradation of the materials.

In this paper, we have investigated the $\mathrm{AlGaN} / \mathrm{GaN}$ heterostructures with two-dimensional electron gas (2DEG), which offer the advantages of a bandgap greater than $2.5 \mathrm{eV}$. This wide bandgap heterostructure offers the advantage of reducing the possibility of thermally induced intrinsic conduction and the existence of a high mobility of a 2-D electron gas layer which greatly enhances the magnetic sensitivity of Hall sensors.

In reported work from another group [6], SPM with AlGaN/GaN-based micro-Hall probes with dimensions

\footnotetext{
Digital Object Identifier 10.1109/TMAG.2008.2001622

${ }^{1}$ Color versions of one or more of the figures in this paper are available online at http://ieeexplore.org.
}

of $2 \times 2 \mu \mathrm{m}$ has been demonstrated using scanning tunneling-guided (STM) feedback at higher temperatures $(\sim 375 \mathrm{~K})$. In this paper, fabrication and characterization of $\mathrm{AlGaN} / \mathrm{GaN}$ heterostructures with dimension of $1 \times 1 \mu \mathrm{m}$ is presented, together with the integration of sensors to the quartz Crystal tuning forks. Although there are other methods like integrating the Hall sensor with piezoresistive [8] and $\mathrm{Si}_{3} \mathrm{~N}_{4}$ atomic force microscopy (AFM) cantilevers [9], it is relatively difficult and cumbersome to microfabricate these sensors. Quartz crystal force sensors [10] have been used in scanning probe microscopy (SPM) for a wide range of applications, since the quartz crystal tuning forks are very cheap, readily available, and the force sensing is performed using a simple current to voltage converter. By using QTF AFM feedback, we have also eliminated the necessity to have a conductive sample or coat the insulating samples with a thin layer of gold. Moreover, the use of gold thin film as an integrated STM tip is also eliminated. On rough surfaces this STM tip can wear off easily and damage to Hall sensor follows inevitably. In this paper, the frequency response of the QTF has been studied in a systematic way based on theoretical models, simulation results, and experimental data. The quartz crystal AFM feedback SHPM is very simple to run, as it uses self-sensing quartz force sensors. The reliable operation of the scanning Hall probe microscopy using the QTF in a wide temperature range of $4.2 \mathrm{~K}-425 \mathrm{~K}$, with a better lifetime performance for the Hall sensor than STM tracking is also demonstrated for the first time.

\section{FABRICATION AND CHARACTERIZATION}

\section{A. Fabrication}

1) Wafer Specifications: The semiconductor wafers used are rotating disc metal-organic chemical vapor deposition (MOCVD) grown $\mathrm{AlGaN} / \mathrm{GaN}$ on $\mathrm{Si}$ (111) 2-D electron gas 


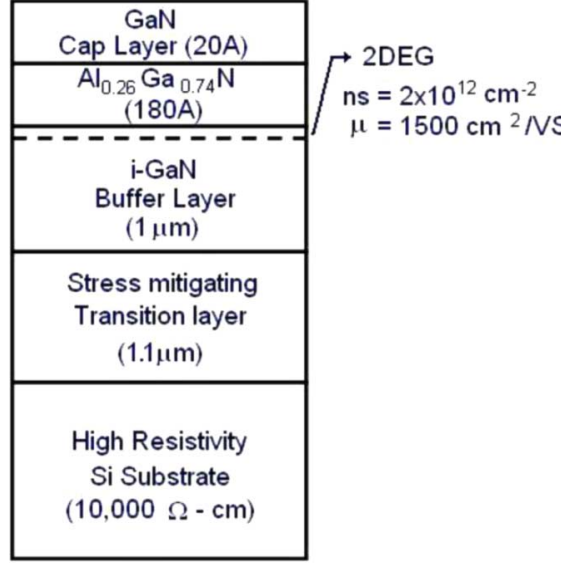

(a)

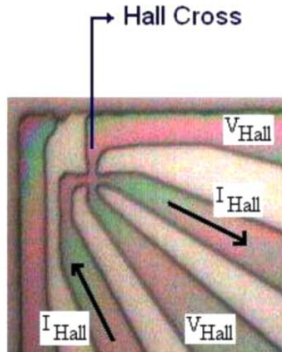

(b)

Fig. 1. (a) Schematic diagram of the layer configuration of the $\mathrm{AlGaN} / \mathrm{GaN}$ heterostructure used. (b) Photograph of $1 \times 1-\mu \mathrm{m}$ Hall probe showing the STM.

(2DEG) material [11]. The epistructure of the wafer, as shown in Fig. 1(a), consists of the following layers; 1) a $20-\AA$-thin layer of GaN cap layer for protection purposes; 2) $180 \AA$ of $\mathrm{Al}_{0.26} \mathrm{Ga}_{0.74} \mathrm{~N}$ layer; 3) 1- $\mu \mathrm{m}$-thick layer of undoped $\mathrm{GaN}$, which forms a $2 \mathrm{DEG}$ at the AlGaN interface; 4) proprietary stress mitigating transition layer of $1.1 \mu \mathrm{m}$; and 5) high resistivity $\mathrm{Si}$ (111) substrate with a resistivity of $10 \mathrm{k} \Omega \mathrm{cm}$. The room temperature sheet carrier concentration and electron mobility of the 2DEG induced at the heterointerface were $2 \times 10^{12} \mathrm{~cm}^{-2}$ and $1500 \mathrm{~cm}^{2} / \mathrm{Vs}$, respectively.

2) Device Fabrication: Micro-Hall probes with effective dimension of $1 \times 1 \mu \mathrm{m}$ have been fabricated using optical lithography in a class 100 clean room environment. Device fabrication process consists of three major steps which are: 1) formation of the mesa and active "cross" patterns by reactive ion etching (RIE) using $\mathrm{CCl}_{2} \mathrm{~F}_{2}$ gas plasma; 2) thermal evaporation of Ti/Al/Ti/Au Ohmic contacts; and 3) rapid thermal processing (RTP) in a nitrogen atmosphere at $850{ }^{\circ} \mathrm{C}$ for $30 \mathrm{~s}$. Four Hall sensors are microfabricated on a $5 \times 5-\mathrm{mm}$ chip at a time and they are diced to a size of $1 \times 1 \times 0.5 \mathrm{~mm}$. The SEM graph of the fabricated Hall probe is shown in Fig. 1(b).

\section{B. Electrical and Magnetic Characterization}

1) Electrical Characteristics: In order to investigate the electrical characteristics of Hall probes, an electrical connection has been established with $12-\mu \mathrm{m}$ gold wire using an ultrasonic wedge bonder. Hall sensors have been characterized based on Hall voltage $\left(\mathrm{V}_{\mathrm{H}}\right)$ versus Hall current $\left(\mathrm{I}_{\mathrm{H}}\right)$ under different bias temperature conditions. As shown in Fig. 2, linear relations can be observed between $V_{H}$ versus $I_{H}$ characteristics with two different dynamic resistances (slope of $\mathrm{V}_{\mathrm{H}}$ versus $\mathrm{I}_{\mathrm{H}}$ curve, $\mathrm{r}_{\mathrm{H}} \mathrm{V}_{\mathrm{H}} / \mathrm{I}_{\mathrm{H}}$ ) values. At $300 \mathrm{~K}$ as shown in Fig. 3, low current regime $\left(\mathrm{I}_{\mathrm{H}}<100 \mu \mathrm{A}\right) \mathrm{r}_{\mathrm{HLC}}$ is $510 \Omega$ and in high current regime $\left(\mathrm{I}_{\mathrm{H}}>100 \mu \mathrm{A}\right)$ it is found to be $\mathrm{r}_{\mathrm{HHC}}=62 \Omega$. It is speculated that this decrease in the $r_{H}$ value is due to the fact that there might be an opening of new conduction channels by applying high current causing an increase in the number of parallel paths. This has been further investigated by increasing the temperature.

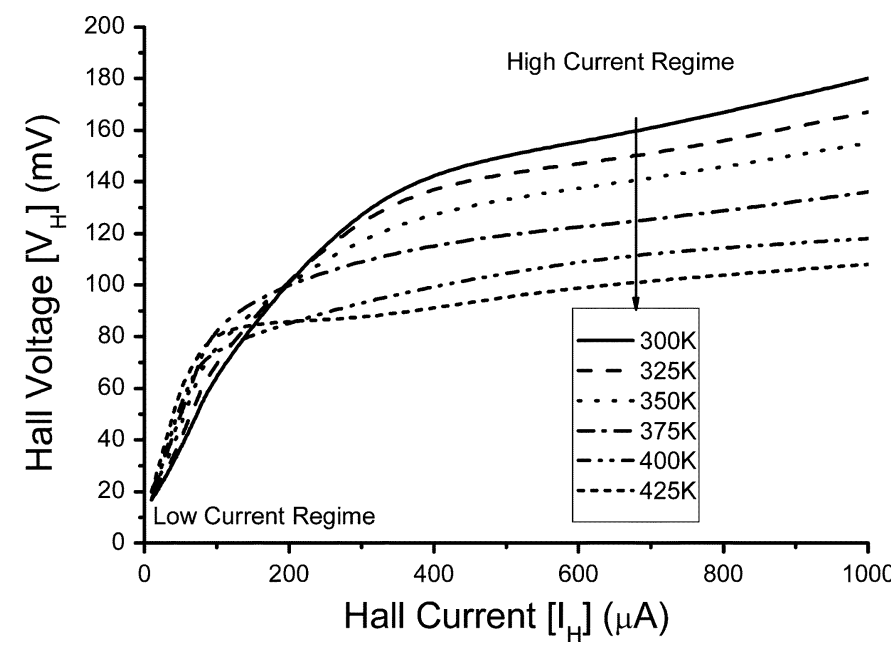

Fig. 2. Effect of temperature on the Hall voltage versus Hall current characteristics.

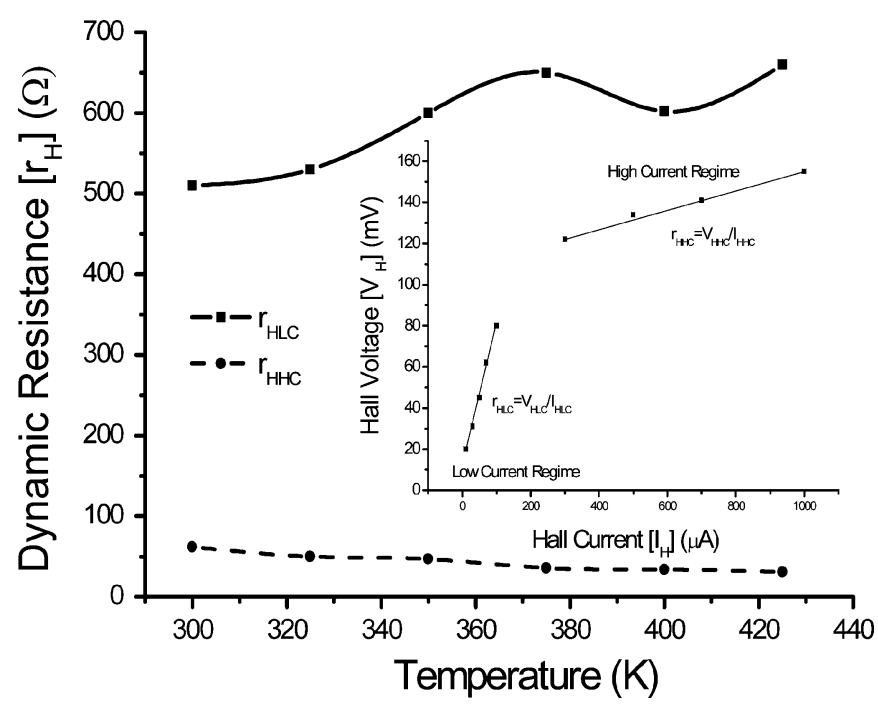

Fig. 3. Effect of temperature on the dynamic resistances $\mathbf{r}_{\mathrm{HLC}}$ and $\mathbf{r}_{\mathrm{HHC}}$. The $\mathbf{r}_{\mathrm{HLC}}$ and $\mathbf{r}_{\mathrm{HHC}}$ are defined in the inset graph.

An increase in temperature from $300 \mathrm{~K}$ to $425 \mathrm{~K}$ shows that $\mathrm{r}_{\mathrm{HLC}}$ increases by $29.4 \%$ and $\mathrm{r}_{\mathrm{HHC}}$ decreases by $50 \%$, which further supports the previously stated argument of increase in the number of channels by increasing Hall current.

2) Magnetic Characterization: Hall probes have been also characterized for their magnetic response by applying magnetic field with an external coil and by measuring the Hall voltage to calculate the value of Hall coefficient, $\mathrm{R}_{\mathrm{H}}$. As shown in Fig. 4, the value of $R_{H}$ also depends on the Hall current and two regimes can be formed as in the case of $\mathrm{V}_{\mathrm{H}}$ versus $\mathrm{I}_{\mathrm{H}}$. At high Hall current bias, $R_{H}$ is more affected by the temperature.

During the experiments, the scanning sensor remains at high temperatures for long periods of time. We have investigated run time effect on $V_{H}$ versus $I_{H}$ and $R_{H}$ versus $I_{H}$ characteristics under high temperature environments. The results showed no significant change in the values, suggesting a safe use of these Hall probes in scanning systems over a long time in harsh conditions. 


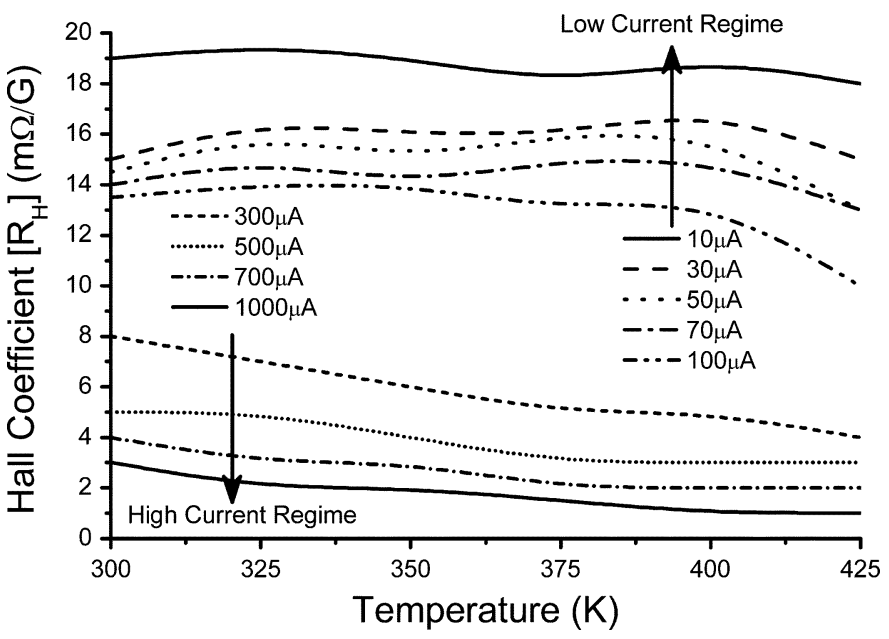

Fig. 4. Effect of Hall current on Hall coefficient versus temperature characterization.

\section{SHPM}

Our SHPM can operate under two different feedback schemes, namely, STM and AFM. As mentioned in Section I, in order to compensate for the drawbacks of STM feedback, especially at high temperatures, a novel method of QTF AFM feedback has been implemented.

\section{A. QTF Hall Probe Microscope System}

In this paper, $32.768 \mathrm{k} \mathrm{Hz}$ quartz crystals tuning forks with dimensions $(1 \times \mathrm{w} \times \mathrm{t})$ of prongs $3.81 \times 0.34 \times 0.62 \mathrm{~mm}$ resulting in stiffness of $29 \mathrm{kN} / \mathrm{m}$ have been used. In order to integrate these force sensors in SHPM for AFM feedback, they are extracted from their cans and their leads have been replaced with a nonmagnetic wiring. Furthermore, these QTFs are glued to a $10 \times 10$-mm printed circuit board sensor holder compatible with the scanning head of the commercially available SHPM system [12]. A Hall probe with chip size of $1 \times 1 \times 0.5 \mathrm{~mm}$ has been mounted on the QTF using low temperature epoxy for low temperature measurement while for high temperature scanning this bond has been reinforced by using super glue. Fig. 5 shows the assembly of the Hall probe on a QTF.

In order to investigate the loading effect of the Hall probe chip on the resonance frequency of the QTF, detailed calculations have been done. These calculations were done based on the two simplest models: simple harmonic oscillator (SHO) and cantilever beam theory (CBT). According to calculations based on these two models, the combination of HP with QTF gives a typical resonance frequency of $31.7 \mathrm{k} \mathrm{Hz}$ ( $\mathrm{SHO}$ model) and $21.2 \mathrm{k} \mathrm{Hz}$ (CBT model). The results are summarized in Table I.

Further, by using the computer simulation program (COSMOS), these QTFs were investigated for different mounting techniques as shown in Fig. 5. It has been observed under unloaded (no Hall probe attached to QTF) conditions when both prongs are free that we have two modes of operation, namely symmetric (both prongs are vibrating up and down in phase) with $16.581 \mathrm{k} \mathrm{Hz}$, (close to CBT model) and Asymmetric (prongs are $180^{\circ}$ out of phase while vibrating up and down) with $31.729 \mathrm{k} \mathrm{Hz}$ (close to SHO). The effect of fixing

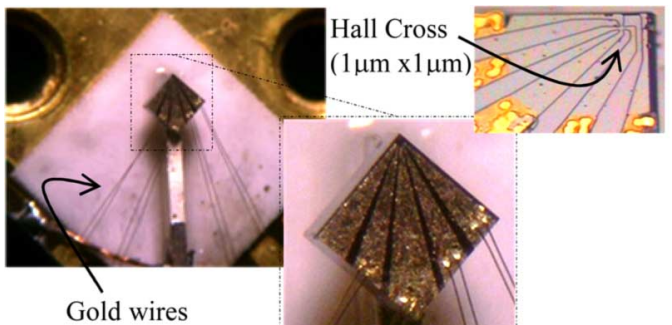

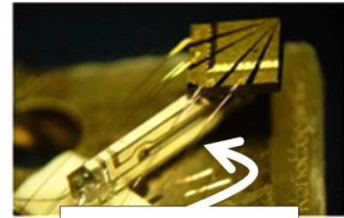

Both prongs free

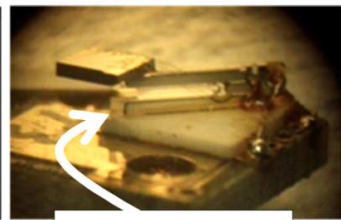

One prong free
Fig. 5. (Top) Integration of Hall probe with QTF with gold wire for electrical contacts. (Bottom) QTF installation techniques, (left) when both prongs are free (free to move) and (right) when bottom prong is fixed to bottom.

TABLE I

CALCUlation Results For QTF

\begin{tabular}{|c|c|c|c|c|}
\hline \multicolumn{5}{|c|}{ Dimensions of Quartz Tuning Fork } \\
\hline \multirow[t]{2}{*}{ Tuning Fork } & Width & Length & Thickness & $\begin{array}{c}\text { Mass of } \\
\text { Cantilever }\end{array}$ \\
\hline & $0.34 \mathrm{~mm}$ & $3.81 \mathrm{~mm}$ & $0.62 \mathrm{~mm}$ & $2.13 \mathrm{mg}$ \\
\hline \multirow[t]{2}{*}{ Hall Probe } & Width & Length & Thickness & $\begin{array}{l}\text { Mass of Hall } \\
\text { Probe }\end{array}$ \\
\hline & $1 \mathrm{~mm}$ & $1 \mathrm{~mm}$ & $0.475 \mathrm{~mm}$ & $1.11 \mathrm{mg}$ \\
\hline \multicolumn{5}{|c|}{ Calculation } \\
\hline $\begin{array}{l}\text { Theoretical } \\
\text { Models }\end{array}$ & \multicolumn{2}{|c|}{$\begin{array}{l}\text { Simple Harmonic } \\
\text { Oscillator }\end{array}$} & \multicolumn{2}{|c|}{ Cantilever Beam theory } \\
\hline $\begin{array}{l}\text { Effective } \\
\text { Mass }\end{array}$ & \multicolumn{2}{|c|}{$\begin{aligned} M_{\text {SHO }} & =\frac{m_{c} M_{t}}{m_{c}+M_{t}} \\
& =0.73 \mathrm{mg}\end{aligned}$} & \multicolumn{2}{|c|}{$\begin{aligned} M_{\text {Cat }} & =M_{t}+0.24 m_{c} \\
& =1.62 \mathrm{mg}\end{aligned}$} \\
\hline $\begin{array}{l}\text { Resonance } \\
\text { Frequency }\end{array}$ & \multicolumn{2}{|c|}{$\begin{aligned} f_{\text {SHO }} & =\frac{1}{2 \pi} \sqrt{\frac{k}{M_{\text {SHO }}}} \\
& =31.7 \mathrm{kHz}\end{aligned}$} & \multicolumn{2}{|c|}{$\begin{aligned} f_{C B T} & =\frac{1}{2 \pi} \sqrt{\frac{k}{M_{C \sigma T}}} \\
& =21.2 \mathrm{kHz}\end{aligned}$} \\
\hline \multicolumn{5}{|c|}{ Simulation } \\
\hline $\begin{array}{l}\text { Vibration } \\
\text { Mode }\end{array}$ & \multicolumn{2}{|c|}{ Symmetric } & \multicolumn{2}{|c|}{ Asymmetric } \\
\hline $\begin{array}{l}\text { Both prongs } \\
\text { are free }\end{array}$ & \multicolumn{2}{|c|}{$17.14 \mathrm{kHz}$} & \multicolumn{2}{|c|}{$31.41 \mathrm{kHz}$} \\
\hline $\begin{array}{l}\text { One prong is } \\
\text { free }\end{array}$ & \multicolumn{2}{|c|}{$17.6 \mathrm{kHz}$} & \multicolumn{2}{|c|}{0} \\
\hline
\end{tabular}

Stiffness Constant $(k)$ was calculated using $3 \frac{E}{l^{3}} \frac{w t^{3}}{12}=2.88 \times 10^{4}[\mathrm{~N} / \mathrm{m}]$

one prong showed up as an elimination of the asymmetric mode and we get a single frequency of $15.642 \mathrm{k} \mathrm{Hz}$.

Simulation results after adding the Hall probe to the QTF show similar results as in the case of the free prong as we can observe the presence of both types of resonance frequencies: $17.138 \mathrm{k} \mathrm{Hz}$ (symmetric mode) and $31.4 \mathrm{k} \mathrm{Hz}$ (asy mmetric mode). Under one-prong-fixed conditions we have a single frequency at $17.6 \mathrm{k} \mathrm{Hz}$. These values are in close agreement with the calculated values from CBT (symmetric mode) and SHO (asymmetric mode) models. The slight discrepancy in the theoretical results, simulation, and given specification is due to the simplicity of the model used where the effect of bonding wires, 


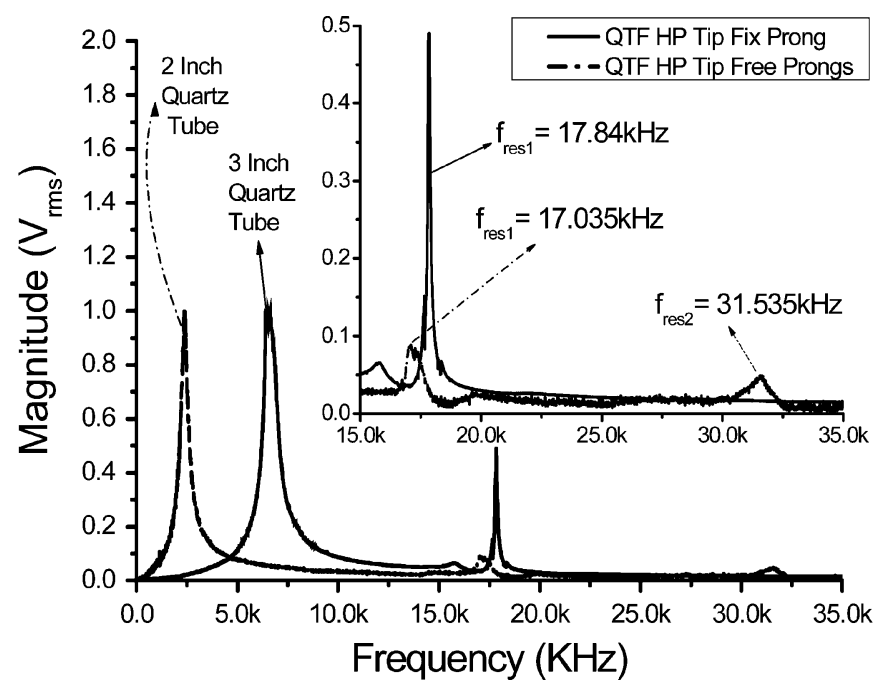

Fig. 6. Frequency response of $32 \mathrm{k} \mathrm{Hz}$ QTF with integrated GaN Hall probe under fixed-prong and free-prong configurations. The effect of the Quartz tube of scan head is also shown.

weight of the glue, and effect of ambient environment has not been included in the calculations.

In order to confirm these results, real probes, where GaN Hall probes have been attached to the tip of the QTF, have been prepared using both configuration (one prong fixed and both prongs free), as shown in Fig. 6. The frequency response of the QTF has been measured using a simple $I-V$ converter with a gain of $1.5 \times 10^{7} \mathrm{~V} / \mathrm{A}$, as we dithered the sensor assembly with the special excitation piezo integrated at the scanner.

From Fig. 6, it is clear that when both prongs are free we have two resonance frequencies around $17.035 \mathrm{k} \mathrm{Hz}$ and $31.535 \mathrm{k} \mathrm{Hz}$, but when we fix one of the prongs we get a single resonance frequency around $17.84 \mathrm{k} \mathrm{Hz}$. A slight discrepancy from the calculated and simulated results is probably due to the mass of glue and the position of the HP has not been included at the calculations and simulation. There is a peak around $\sim 3 \mathrm{k} \mathrm{Hz}$ or $\sim 6 \mathrm{kHz}$, as seen in Fig. 6 . This is due to the resonance frequency of the whole scanner assembly. We have used two different systems with 2- and 3-in-long scan piezo tubes.

Based on the above results, we can conclude that for stable operation one prong of the TF should be fixed. Under such conditions we are close to symmetric mode and this can be easily explained by using the cantilever beam theory.

\section{B. Scanning Results and Discussion}

A commercial low-temperature SHPM system [11] with some modifications for high-temperature measurements is used to perform the scanning experiments. The Hall sensor is positioned $12 \mu \mathrm{m}$ away from the corner of a deep etch mesa, which serves as a crude AFM tip. The sample is tilted $\sim 1^{\circ}-2^{\circ}$ with respect to the Hall probe chip, ensuring that the corner of the mesa is the highest point. As the combined sensor approaches the surface, the resonant frequency of the sensor shifts due to tip sample forces. The sensor assembly is dithered at the resonance frequency with the dedicated split section on the scan piezo tube using a digital phase locked loop
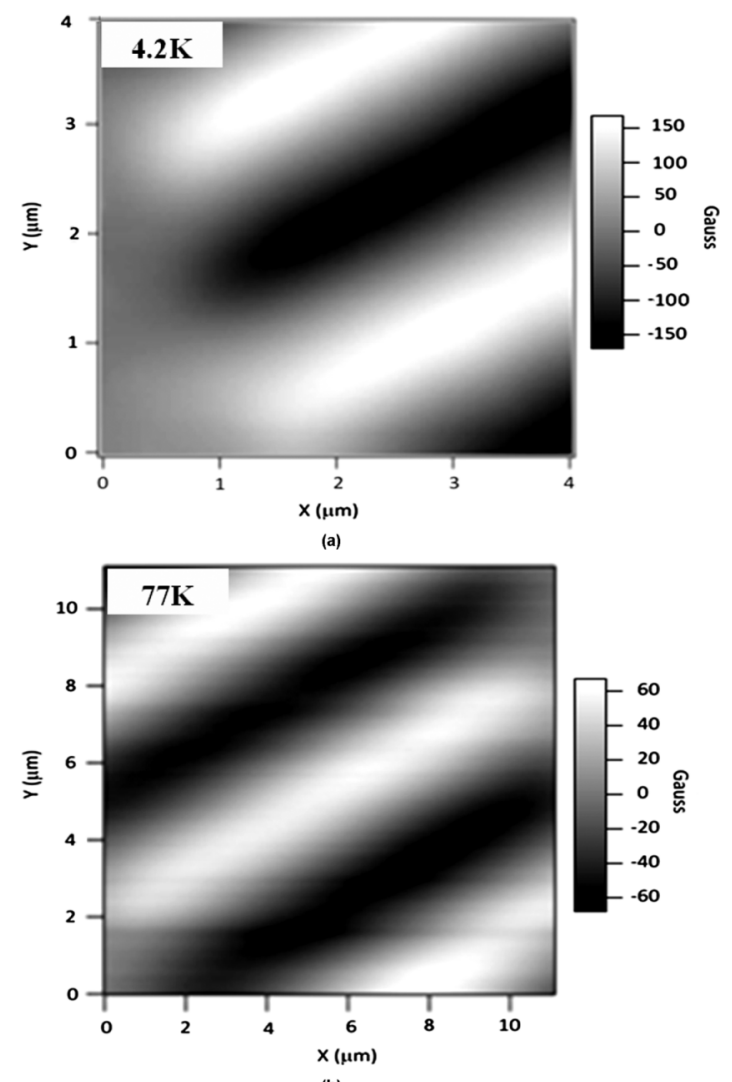

(b)

Fig. 7. SHPM image of hard disk sample at (a) liquid helium temperature $(4.2 \mathrm{~K})$ and (b) liquid nitrogen temperature $(77 \mathrm{~K})$. Scanning speed was $5 \mu \mathrm{m} / \mathrm{s}$.

(PLL) circuit. The frequency shift $\Delta f$, measured by the PLL circuit is used for AFM feedback to keep the sensor sample separation constant with the feedback loop. The microscope can be operated in two modes: AFM tracking and liftoff mode. In our scanning experiments, we have used the AFM tracking mode with a $\Delta f$ (amount of frequency shift) $=10 \mathrm{~Hz}$. Furthermore, in order to detect AFM topography and the error signal generated by the PLL, along with the magnetic field image, SHPM electronics are modified. Even though a relatively heavy mass is attached at the end of the tuning fork, we usually get a quality factor, $Q$, at 150-220, even at atmospheric pressures. Despite more or less the planar geometry, the viscous damping is not a big problem due to high stiffness of the force sensor.

We have imaged magnetic bits and topography of the hard disk at various temperatures to show the performance of the microscope with AFM feedback using QTF. In order to scan at low temperatures, the sample space is filled with 1 Atm of Helium exchange gas at $300 \mathrm{~K}$ and cooled down to $4.2 \mathrm{~K}$. The Hall sensor was driven with $500 \mu \mathrm{A}$ dc current, the series resistance of the Hall sensor was $0.5 \mathrm{k} \Omega, 3 \mathrm{~K} \Omega$, and $15 \mathrm{k} \Omega$ at $4.2 \mathrm{~K}, 77 \mathrm{~K}$, and $300 \mathrm{~K}$, respectively. The resonance frequency and the quality factor were $17780 \mathrm{~Hz}$ and $\mathrm{Q} \sim 60,17630 \mathrm{~Hz}$ and $\mathrm{Q} \sim 97$, and $16944 \mathrm{~Hz}$, and $\mathrm{Q} \sim 305$ at $4.2 \mathrm{~K}, 77 \mathrm{~K}$, and $300 \mathrm{~K}$, respectively. Fig. 7 shows the magnetic image and topography of the hard disk sample obtained in AFM tracking mode at $4.2 \mathrm{~K}, 77 \mathrm{~K}$ with a scanning speed of $5 \mu \mathrm{m} / \mathrm{s}$ and scan area of $17 \times 17 \mu \mathrm{m}$ and $30 \times 30 \mu \mathrm{m}$, respectively. 

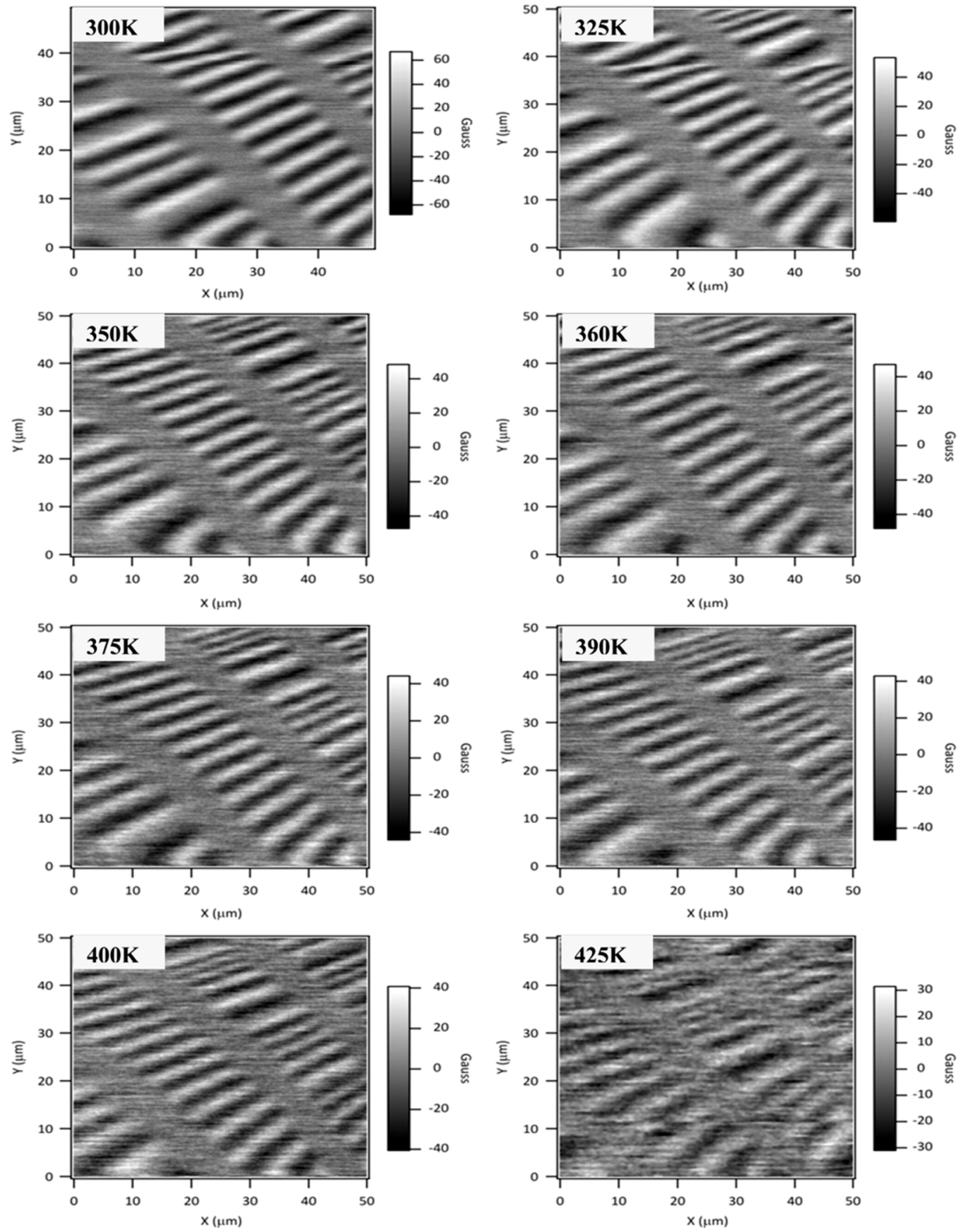

Fig. 8. SHPM image of hard disk sample at high temperatures. Scanning speed was $5 \mu \mathrm{m} / \mathrm{s}$. Distortions at $425 \mathrm{~K}$ are most probably due to loss of adhesion between the HP and tuning fork.

In order to investigate the high-temperature operation of these GaN micro Hall probes, a low noise heater stage was embedded in the LT system. The results shown in Fig. 8 confirm the success of the AFM feedback tracking system with micro-Hall probes up to $425 \mathrm{~K}$. The observed distortions in the scanned images at high temperatures are considered to be not due to performance of the Hall probe, but it is mainly due to the properties of the QTF and the problems related with the used glue and degradation of the sample. The resonance frequency and quality factor of the quartz crystal tuning fork changes with the temperature as shown in Fig. 9; however, this change is still much less than the conventional silicon AFM cantilevers. Further investigation is underway to find out the methods to improve the thermal stability of the QTF AFM feedback and exploration of GaN Hall probe microscopy at even higher temperatures.

\section{CONCLUSION}

Micro-Hall probes with a Hall cross area of $1 \times 1 \mu \mathrm{m}$ with $\mathrm{AlGaN} / \mathrm{GaN}$ heterostructure were fabricated and characterized for their application in variable temperature magnetic imaging. Electrical and magnetic characteristics can be divided into two regimes based on the Hall current value. In each regime, Hall 


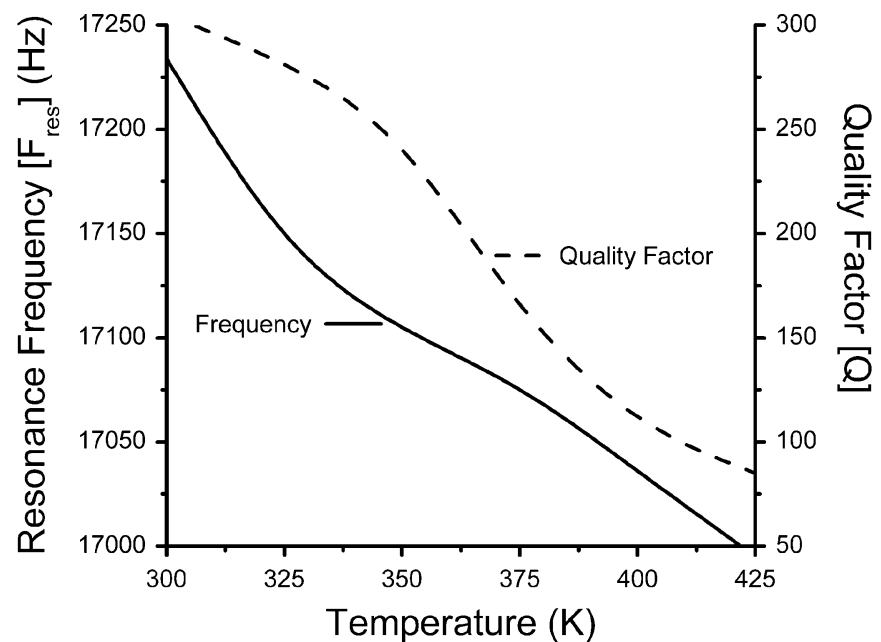

Fig. 9. Effect of working temperature on the resonance frequency and quality factor of the QTF.

voltage versus Hall current characteristics showed linear behavior, while the slope of $\mathrm{V}_{\mathrm{H}}$ versus $\mathrm{I}_{\mathrm{H}}$ curve (dynamic resistance) was high in the low current regime and low in the high current regime. It was also a function of working temperature. The low current regime shows less effect on $V_{H}$ curves and has high $R_{H}$ value but a value of $V_{H}$ is low. These Hall probes have been integrated with a QTF and successfully used in scanning Hall probe microscopy. Simulation and experimental study of the QTF with Hall sensor shows that the system works better if one prong of the QTF is fixed. SHPM using a Quartz crystal tuning fork AFM feedback with $1 \times 1-\mu \mathrm{m} \mathrm{AlGaN/GaN}$ Hall probes has been successfully demonstrated for a hard disk sample for temperature range of $4.2 \mathrm{~K}$ to $425 \mathrm{~K}$. We are continuing our efforts to achieve higher spatial resolution and even higher temperature applications of GaN Hall probes.

\section{ACKNOWLEDGMENT}

This work was supported in part by TüBITAK, Turkey, under Projects TBAG-(105T473) and TBAG-(105T224). The work of
A. Oral was supported by the Turkish Academy of Sciences (TüBA). The authors would like to thank M. Demir for his continuous support and for the upkeep of the microscope.

\section{REFERENCES}

[1] A. Sandhu, K. Kurosawa, M. Dede, and A. Oral, "50 nm Hall sensors for room temperature scanning Hall probe microscopy," J. Appl. Phys, vol. 43, pp. 777-778, 2004.

[2] J. R. Kirtley and J. P. Wikswo, "Scanning SQUID microscopy," Аnnu. Rev. Mater. Sci., vol. 29, pp. 117-148, 1999.

[3] Y. Martin and H. K. Wickramasinghe, "Magnetic imaging by "force microscopy with 1000 Å resolution"," Appl. Phys. Lett., vol. 50, pp. 1455-1457, 1987.

[4] E. Betzig, J. K. Trautman, R. Wolfe, E. M. Gyorgy, P. L. Finn, M. H. Kryder, and C. H. Chang, "Near-field magneto-optics and high density storage," Appl. Phys. Lett., vol. 61, pp. 142-145, 1992.

[5] F. Schmidt and A. Hubert, "Domain observations on CoCr-layers with a digitally enhanced Kerr-microscope," J. Mag. Magn. Mater., vol. 61, pp. 307-320, 1986.

[6] Z. Primadani, H. Osawa, and A. Sandhu, "High temperature scanning Hall probe microscopy using $\mathrm{AlGaN} / \mathrm{GaN}$ two dimensional electron gas micro-Hall probes," J. Appl. Phys., vol. 101, pp. 09K105-09K105-3, 2007.

[7] T. Yamamura, D. Nakamura, M. Higashiwaki, T. Matsui, and A. Sandhu, "High sensitivity and quantitative magnetic field measurements at $600^{\circ}$ C," J. Appl. Phys., vol. 99, pp. 08B302-08B302-3, 2006.

[8] A. J. Brook, S. J. Bending, J. Pinto, A. Oral, D. Ritchie, H. Beere, M. Henini, and A. Spring Thorpe, "Integrated piezoresistive sensors for atomic force-guided scanning Hall probe microscopy," Appl. Phys. Lett., vol. 82, pp. 3538-3540, 2003.

[9] B. K. Chong, H. Zhou, G. Mills, L. Donaldson, and J. M. R. Weaver, "Scanning Hall probe microscopy on an atomic force microscope tip," J. Vac. Sci. Technol. A, vol. 19, pp. 1769-1772, 2001.

[10] P. Guethner, U. Fischer, and K. Dransfeld, "Scanning near-field acoustic microscopy," Appl. Phys. B, vol. 48, pp. 89-92, 1989.

[11] P. Rajagopal, T. Gehrke, J. C. Roberts, J. D. Brown, T. Warren Weeks, E. L. Piner, and K. J. Linthicum, "Large-area, device quality GaN on Si using a novel transition layer scheme," in Proc. Material Research Soc. Symp., 2003, vol. 743, no. 3.

[12] Low temperature scanning hall probe microscope (LT-SHPM) [Online]. Available: www.nanomagnetics-inst.com NanoMagnetics Instruments Ltd., Oxford, U.K.

Manuscript received February 24, 2008. Current version published December 17, 2008. Corresponding author: R. A. Akram (e-mail: rizwanakram75@gmail. com). 